\title{
Medical and Interventional Treatment of Refractory Angina
}

\author{
Valchanov $\mathrm{K}^{1 *}$, Tan $\mathbf{Z}^{1}$ and Valchanova $\mathbf{A}^{\mathbf{2}}$ \\ ${ }^{1}$ Department of Anaesthesia, Royal Columbian Hospital, \\ 330 E Columbia St, New Westminster, Canada \\ ${ }^{2}$ Student at University College London, London, UK \\ *Corresponding author: Kamen Valchanov, \\ Department of Anaesthesia, Royal Columbian Hospital, \\ 330 E Columbia St, New Westminster, BC V3L 3W7, \\ Canada
}

Received: September 08, 2021; Accepted: October 08, 2021; Published: October 15, 2021

\begin{abstract}
Refractory Angina Pectoris is a chronic pain condition relating to myocardial ischaemia in the presence of coronary artery disease despite optimal medical therapy. It affects a cohort of patients with a subset of the coronary perfusion deficiencies. While there is no cure for these patients, an array of medical and interventional treatment is available in 2021. In this article we discuss the options available with an explanation of the mechanisms of action and evidence for their use.
\end{abstract}

Keywords: Refractory Angina Pectoris; Chronic Coronary Disease; Spinal Cord Stimulation

\section{Introduction}

\section{Definition}

Refractory Angina Pectoris (RAP) is defined as "a chronic condition caused by clinically established reversible myocardial ischaemia in the presence of coronary artery disease, which cannot be adequately controlled by a combination of medical therapy, angioplasty, or coronary artery bypass grafting" [1]. RAP encompasses conditions where the ischaemic pain is due to a deficiency in distal myocardial perfusion such as microvascular obstruction and where patients have already undergone often extensive coronary revascularization both surgically and percutaneously. In many of these patients, further revascularization is not technically possible, or such procedures carry a prohibitive risk.

However, the routine assessment of microvascular obstruction is both technically challenging and infrequently performed. With recent advances in coronary revascularization, the paradigm of what is not possible or too risky is constantly evolving. This improvement has been arguably most marked in the field of percutaneous coronary intervention for chronic total occlusions. This advance alone has led to a reduction in patients who have 'no option' for further coronary revascularization in 2021. However, a small subset of patients with stable coronary atherosclerosis who have a significant morbidity and reduction in quality of life due to ongoing chest pain still exists.

\section{Prevalence}

Over the last 20 years, significant progress has been made in reducing the mortality in patients suffering with coronary atherosclerosis, whilst the morbidity of patients living with daily stable angina remains a significant burden on the health economy. The prevalence of RAP is around $10 \%$ in patients with chronic stable coronary disease [2].

Although RAP may not, in isolation, result in a substantial reduction in life expectancy, it does frequently impair the quality of life [3]. In a large case series from Duke Database Cardiovascular Disease, patients with RAP have a small increase in mortality rates at 3 years, but a high incidence of resource utilization and hospital admissions [4]. This was costed in 2012 at 10,185 USD per patient. This implication is significant when one considers that $77.6 \%$ of patients are still alive at 9 years [5]. Even 21 years ago the cost of hospitalization of RAP in the UK in 2000 was estimated as $£ 208$ million per year [6].

Many publications have addressed the predictive test for death and further myocardial infarction, and the most useful test associated with these two events is the plasma concentration of hs-cTnT over 5ng/l [7]. Interestingly, a study in the New England Journal of Medicine [8] randomly assigned 5179 patients with moderate to severe coronary ischemia to either conservative management or aggressive revascularisation strategy. As expected, $90 \%$ of the patients had also symptoms of angina. The authors' findings were that at 3.2 years both groups had similar results in terms of mortality. The health status of the same group of patients in the ISCHEMIA trial [8] showed also similar results in both groups, but better anginal control in the invasive strategy group. These results support the hypothesis that invasive treatment of coronary obstructive lesions is mandated only in symptomatic patients with angina.

\section{Assessment of RAP}

\section{Clinical history}

A key concept of the management of patients with refractory angina is that the chest pain they are experiencing is, indeed, caused by an insufficiency in myocardial oxygen delivery. We, as clinicians, are often surprised by the enormous variation in perceived pain by patients suffering with myocardial ischaemia, most notably in those patients with end organ signs of neuropathy. The diagnosis of anginal pain is in the history. Description of the type of pain (heaviness, gripping, suffocating), location and radiation, provoking and relieving factors, duration and frequency form the basis underlying a definitive diagnosis. However, typical angina is considered to be an uncommon presentation [9]. The perceived pain of refractory angina is worse in patients suffering from anxiety and depression [10]. With time and treatment, the pain is associated with lower emotional arousal. However, as a chronic pain of more than 3 months duration, there is undoubtedly a superimposed emotional and psychosocial 
component. Therefore, in the management of refractory angina, it is equally important to consider a holistic approach to pain, rather than solely concentrate on myocardial oxygen delivery.

The ESC 2019 Guidelines [11] place some emphasis on the holistic approach to the assessment of angina with a screen for noncardiac vascular disease, arrythmias, valvular or hypertrophic cardiac disease and significant other co-morbidities such as thyroid disease, anaemia and renal dysfunction.

\section{Investigations}

The route which the physician may choose for investigation of RAP would very much depend on the pre-test probability calculated on an individual basis for each patient. The use of computed tomography coronary angiography has a prominent role in the new ESC guidelines [11], alongside functional assessments of myocardial ischaemia such as stress echocardiography, cardiac magnetic resonance imaging and radioisotope based myocardial perfusion scanning. If a patient has a high pre-test probability of obstructive coronary disease, then the first line investigation may well be invasive coronary angiography, especially in the presence of reduced left ventricular function. Invasive angiography may also be more useful in patients with arrhythmias which can result in poor gating of the CTCA, or in cases where the likelihood of a high coronary calcium score making accurate luminal assessment difficult. The ESC guidelines also give due prominence to the Fractional Flow Reserve (FFR) assessment of indeterminate lesions and encourage pressure wire assessment in all patients with multi-vessel disease prior to referral for surgical revascularisation

\section{Diagnosis and syndromes}

RAP pain as a condition is caused by the deficiency of oxygen delivery to the cardiomyocytes. RAP, therefore, requires viable myocardium. Areas where cardiomyocytes are replaced by fibrosis are unlikely to produce ischaemic pain. In other cases, a typical anginal pain is present but no epicardial structural cause for the ischaemia can be found. As discussed, these patients may be suffering with undetected microvascular obstruction, or indeed the presence of vasospastic coronary reactivity which can be refractory to optimal vasodilatation with calcium channel blockers and longacting nitrates. A further subset of this population are those patients labelled with cardiac syndrome $\mathrm{X}$, who have unobstructed epicardial coronaries but objective evidence of ischaemia on exercise testing [12]. The hypothesised mechanisms underlying this condition include undiagnosed microvascular obstruction and an abnormal pain perception from the myocardium. Up to $20 \%$ of patients complain from angina but have no angiographic stenosis [13]. How can pain be explained in these patients? It is stipulated that angina can be explained by very high or low coronary flow reserve; diffuse atherosclerosis; heterogeneous endothelial dysfunction; or diffuse epicardial atherosclerosis. The important phenomenon of subendocardial ischaemia [14] which occurs due to increased coronary pressure gradient in periods of increased demand has been widely recognised. These patients may not have angiographically diagnosed flow obstruction.

The recently published European Society of Cardiology guidelines (2019) [11] on Chronic Coronary syndromes, outline a six-step approach for those patients with angina and suspected Coronary Artery Disease (CAD). Thereafter, lifestyle interventions, drug therapy and revascularisation options are considered. Emphasis is placed on individual risk/benefit analysis and a multi-disciplinary approach to revascularisation recommendations.

\section{Management of RAP (Figure 1) \\ Medical therapy}

The standard pharmacological interventions for angina still consist of beta-blockers and calcium antagonists as first line medication. Nitrates are now second line treatments, together with ivabradine, ranolazine and nicorandil.

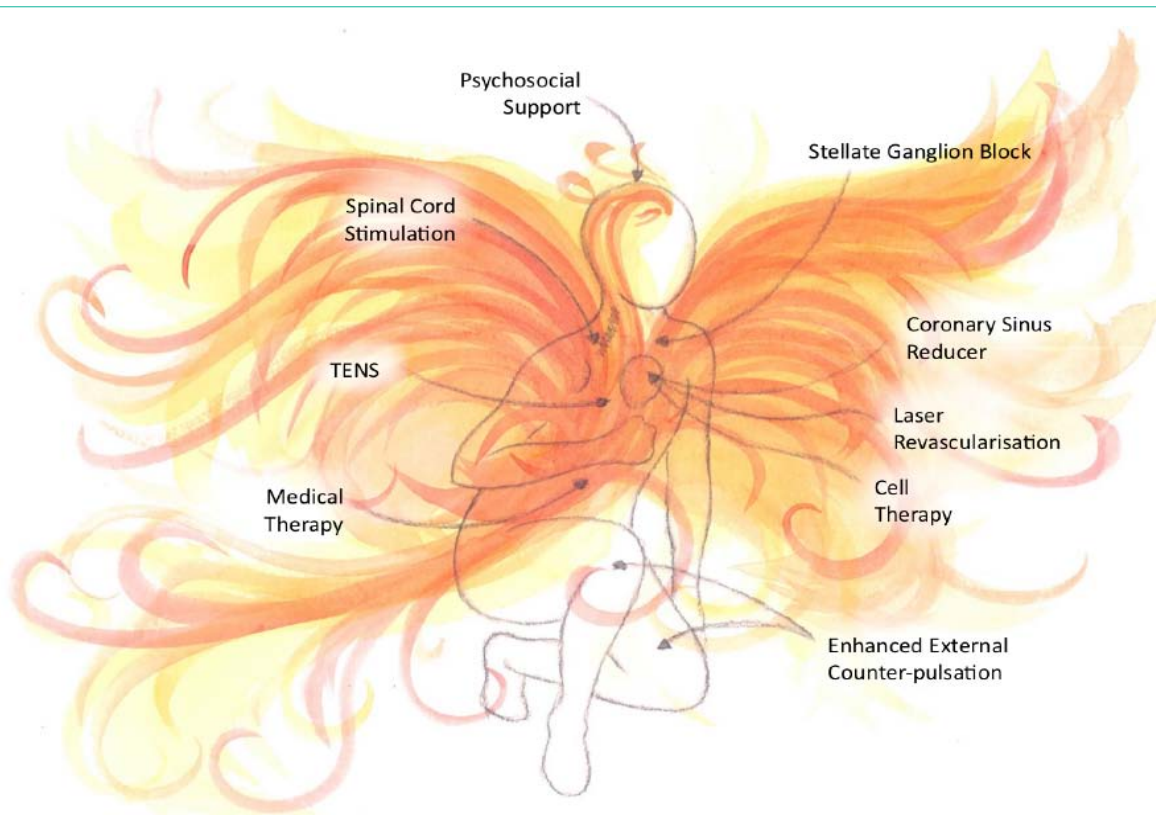

Figure 1: Refractory Angina Pectoris Treatments. Like a Phoenix, the patients with Refractory Angina Pectoris do not die, they are rather revitalized by successful treatments of their chronic pain condition. The diagram illustrates the variety of treatment options available for patients with Refractory Angina Pectoris. 
Ranolazine is a piperazine derivate that inhibits sodium and potassium channels thereby increasing cytosolic calcium concentrations but it does not affect pulse rate or blood pressure. A COCHRANE review of 17 RCT's found that a dose of $1000 \mathrm{mg}$ twice a day as add-on treatment reduced the severity of angina pectoris. It was however associated with increased risk of non-severe adverse effects [15]. The current recommended doses for ranolazine are $375 \mathrm{mg}$ po bd, with a gradual titration to $500 \mathrm{mg}$ po bd and then $750 \mathrm{mg}$ po bd if tolerated. In a recent trial, it was also shown to reduce patient's interaction with the healthcare system [16].

Greater prominence has also recently been given to the addition of a second anti-platelet drug to aspirin when the relative risk of bleeding is outweighed by the ongoing risk of a myocardial event. The ESC has also recommended that low-density lipoprotein levels be reduced below $1.4 \mathrm{mmol} / \mathrm{L}$ in patients with ischaemic heart disease [17].

\section{Coronary revascularisation approach}

The role of a multi-disciplinary revascularisation team is key to the successful management of patients with RAP. Careful counselling and outlining of all the various risks and benefits of both revascularisation strategies is important when involving the patient in decision making. Many patients with RAP will have concomitant reduction in left ventricular ejection fraction on transthoracic echocardiogram making open surgeries riskier. Furthermore, a subgroup of these patients will already have undergone coronary artery bypass grafting, making redo surgery both technically challenging and of higher inherent risk. This danger is largely driven by the presence of a patent left internal mammary graft and the possibility of damage during redo sternotomy.

The percutaneous technologies now available have widened the portfolio of potentially treatable disease within the coronary tree. The recent advances in chronic total occlusion [18] therapy, and the use of intravascular lithotripsy are two examples of technological advances that have allowed greater consideration of revascularisation in patients in whom there would historically have been no option.

\section{Transcutaneous electrical nerve stimulation (TENS)}

TENS has been used for treatment of RAP since the 1980s [19]. It is thought to improve pain control in these patients by utilizing the Gate Theory hypothesized by Melzack and Wall. It was initially presumed that the benefit from the TENS machine for RAP were explained by improving the collateral blood flow in the myocardium. Investigations attempting to prove that it also improved coronary blood flow during the periods of stimulation have failed. Hallen et al demonstrated that using TENS machine produces vasodilatation in the forearm of healthy volunteers but not in RAP sufferers [20]. Presumably TENS therefore exerts its analgesic effects for these patients by another mechanism. However, TENS is used as an assessment tool to predict whether spinal cord stimulation is likely to produce analgesic benefit. The biggest problem with using TENS for RAP sufferers is that it requires time to apply the electrodes to the skin, attach them to the pulse generator before initiation of therapy. As RAP can occur at any time, it is not always a practical solution. Many patients are advised to use TENS frequently throughout the day and prior to an activity which is likely to bring about anginal pain.

\section{Cardiac sympathectomy}

It has long been postulated that sympathetic hyperactivity produces coronary constriction and therefore blocking the sympathetic efferent would improve coronary flow. A recent analysis of all published 528 cases of surgical sympathectomy as a treatment for RAP provided no evidence for symptomatic or quality of life improvement [21].

An easier access to sympathetic fibers responsible for the cardiac plexus is the stellate ganglion at C6 cervical vertebra. It has been well documented that Left Stellate Ganglion Block (LSGB) reliably dilates the left internal mammary artery. Randomised controlled trials and numerous case series have demonstrated the efficacy of this method [22]. Nevertheless, the question on why temporary nerve blockade with local anaesthetic for few hours could produce improved anginal control for weeks and months remains. Secondly, it is unclear, and under investigated, as to why left rather than right stellate ganglion block produces analgesic benefit. Finally, there is no data on the permanent effects of multiple repeated stellate ganglion blocks as well as long term consequence of the complication of Horner's syndrome.

\section{Spinal cord stimulation (SCS)}

SCS has been a routine therapy for pain control in RAP patients. Several clinical trials [23] have addressed the effectiveness of this treatment for the last 30 years. Large case series [24] and metaanalysis [25] have also been published recently. While the mechanisms of how this treatment improves RAP pain control is not entirely clear, the evidence strongly suggests that it improves the anginal class and quality of life. The ESC recommends this treatment as a part of the armoury for RAP patients with category B and C evidence [26]. The hardware of SCS includes an epidural electrode in high thoracic and low cervical area (Figure 2) connected to an electrical generator. While the systems are expensive, the complication rate is low and acceptable as it reduces the frequency of repeated visits to the emergency department of patients with RAP.

While newer modes of SCS like 'burst stimulation', predominantly targeting dorsal columns, are used frequently in the general pain population, there is no clear evidence whether these "paraesthesia free" modes are superior for RAP sufferers [27]. Nevertheless, the scientific explanations of these modes of neurostimulation render it advantageous for patients on whom psychological components of anginal pain are predominant.

The evidence from randomised controlled trials consistently demonstrates that SCS improves the symptoms of RAP as well as the quality of life, as demonstrated by SF-36 and SAQ questionnaires.

\section{Psychosocial support}

RAP is a chronic pain condition. It therefore requires a complex, multimodality approach in the therapeutic management. Mere pharmacological and interventional treatments are only the basic requirements. The complexities of disability and unemployment as well as psychosocial support mandate that these patients are managed by multitude of healthcare professionals. A central tenet of this management is trust. Many patients who have already had surgical and percutaneous interventions are believed to have received the best treatment and assumed to be pain free. Establishing rapport between care givers and patients and their family is the first step to help. Impact 


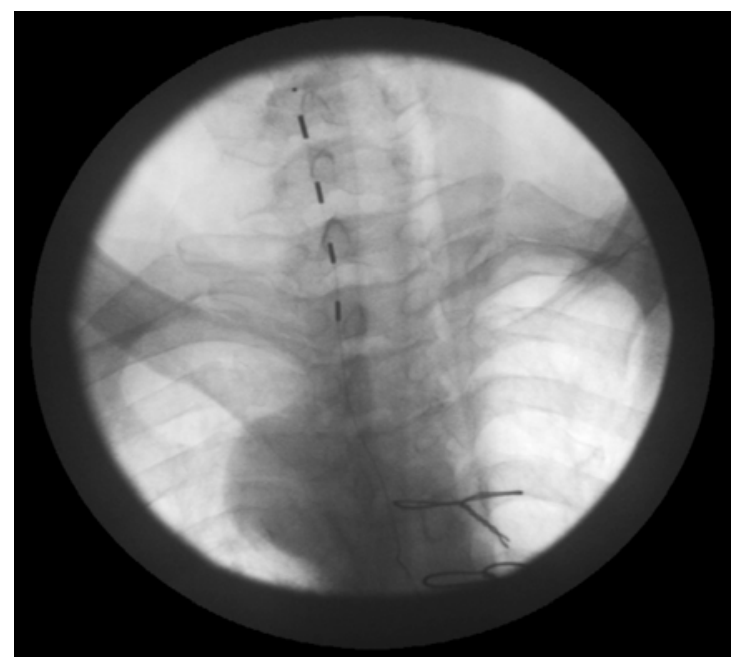

Figure 2: Spinal Cord Stimulator lead at the level of C6 vertebra.

of psychosocial support on RAP patients is difficult to express in numbers. Few studies have managed. Ashbury et al. [28] randomised 42 patients to 8 weeks cardiac rehabilitation programme or symptom diary control. Despite unchanged anginal frequency and severity they found that the interventions not only improved physical ability but also improved psychological wellbeing, as measured by Health Anxiety Questionnaire and York Beliefs Anginal Treat Perception.

Cognitive behavioural therapy is well recognised in the management of chronic pain conditions. Cognitive appraisal of irrational thought like catastrophising and overgeneralization have been used. Strategies for prioritizing, time management, establishing personal values, and avoiding stress-inducing situations have been proven beneficial. While it has not been tested on its own for RAP patients, its role in cardiac rehabilitation is proven. Finally, cognitive behavioural interventions have been found to reduce the number of hospital admissions among patients suffering from RAP [29].

\section{Enhanced external counter pulsation}

Enhanced external counter pulsation has been in use for a long time for variety of conditions, including acute myocardial infarction, cardiogenic shock, and congestive heart failure. Three sets of pneumatic devices are mounted on the lower limbs and inflated sequentially in diastole to $260 \mathrm{mmHg}$ for 60 minutes 5 days a week for 7 weeks. Few studies demonstrated reduction in CCA class, symptoms of depression in up to 1 year, and reduction in healthcare costs [30] and increased markers of angiogenesis [31]. The proposed mechanism of action is increase in pre-load during treatment and hence increase in cardiac output by Starling mechanism. It has also been shown to improve endothelial function, reduce inflammatory cytokines, promote vascular tone, decrease oxidative stress and atherosclerosis, recruit and develop coronary collaterals.

\section{Laser revascularization}

The technique of laser myocardial revascularisation involves drilling full thickness channels in ischaemic myocardium from the endocardial or epicardial site in an attempt to improve the entry of erythrocytes into the myocardium and hence improve oxygen delivery. Despite initial encouraging results the later studies highlighted the role of placebo effect with this technique. The 2017 COCHRANE review found that the risks associated with this technique overweighed the benefits [32].

\section{Coronary Sinus Reducer}

A coronary sinus reducer is an endovascular, balloon expandable, stainless steel, hour-glass shaped device. It produces focal narrowing of the coronary sinus thereby increasing pressure in the coronary venous system and arteriolar dilatation, and hence improvement of epi- and endocardial blood flow leading to anginal relief. The proposed mechanism involves recruitment of collateral coronary flow and redistribution from less-ischaemic endocardium to moreischaemic endocardium. In several randomised trials it reduced the CCA anginal score and Seattle Angina Questionnaire [33,34]. It did not statistically improve exercise duration, time to ST-segment depression or wall-motion index.

\section{Stem cell therapy}

What is stem cell therapy? Several vascular specific growth factors (like vascular endothelial growth factor A VEGF-A) have been translocated via adenovirus vector transfer in a hope to improve myocardial function and symptoms. This proangiogenic gene is hoped to promote neo-revascularisation. Several studies have been performed to assess the effectiveness of this strategy. The RENEW trial demonstrated improved anginal symptoms at 6 months post intramyocardial autologous CD34 cell administration for RAP patients [35]. However, due to its early termination by the sponsor, it could not produce sufficient data to support this treatment as beneficial in the future. Similarly, in a small trial of 31 patients the REGENTVSEL trial of bone marrow derived CD133 cells intramyocardially did not show a benefit for RAP patients. Overall, the meta-analysis by Shah et al. [36] found that in all available 10 trials with total of 648 patients the stem cell therapy improves pain control, as documented in reduction of CCA class, exercise capacity, and improved ejection fraction. Cell therapy is promising future treatment for refractory angina patients [37] as it represents a section of medicine developing very fast. 


\section{Conclusion}

Refractory angina pectoris is a common disease in the patient with coronary atherosclerosis and is often sub optimally managed. As the prevalence of ischaemic heart disease increases, and the population ages, we are likely to see many more survivors of acute cardiac ischaemia who progress to this chronic and disabling cardiac condition. The healthcare burden of this population is likely to significantly increase. Novel revascularisation strategies, together with adjunctive therapies allowing neuromodulation and the reduction in myocardial ischaemia, hold significant potential for the amelioration of the morbidity impact of this condition.

The multi-disciplinary approach to patients with RAP cannot be over-emphasized to achieve the best possible result for these patients. Further work needs to be undertaken to better comprehend the full epidemiological extent of this condition, and to more rigorously evaluate the role of microvascular obstruction in patients with chest pain and grossly unobstructed epicardial coronaries. Our 'no-option' patients deserve a thorough assessment and strategic formulation to enable them to live a better life alongside their chronic cardiac condition.

\section{References}

1. Mannheimer C, Camici P, Chester MR, Collins A, DeJongste M, et al. The problem of chronic refractory angina. Report from the ESC Joint Study Group on the treatment of refractory angina. Eur Heart J. 2002; 23: 355-370.

2. Gallone G, Baldetti L, Tzanis G, Gramengna M, Latib A, Colombo A, et al. Refractory Angina: From Pathophysiology to New Therapeutic Nonpharmacological Technologies. J Am Coll Cardiol Intv. 2020; 13: 1-19.

3. Cheng K, Sainsbury $P$, Fisher M, de Silva R. Management of refractory angina pectoris. European Cardiology Review. 2016; 11: 69-76.

4. Povsic TJ, Broderick S, Anstrom KJ, Shaw LK, Ohman EM, Eisenstein EL, et al. Predictors of long-term clinical endpoints in patients with refractory angina. Journal of the American Heart Association. 2015; 4.

5. Haase R, Schlattmann P, Gueret P, Andreini D, Pontone G, Alkadhi H, et al. Diagnosis of obstructive coronary artery disease using computed tomography angiography in patients with stable chest pain depending on clinical probability and in clinically important subgroups: Meta-analysis of individual patient data. The BMJ. 2019; 365.

6. Stewart S, Murphy N, Walker A, McGuire A, McMurray JJV. The current cost of angina pectoris to the National Health Service in the UK. Heart. 2003; 89: 848-853.

7. Poppi NT, Gowdak LHW, Dourado LOC, Adam EL, Leite TNP, Mioto BM, et al. A prospective study of patients with refractory angina: outcomes and the role of high-sensitivity troponin T. Clinical Cardiology. 2017; 40: 11-17.

8. Maron DJ, Hochman JS, Reynolds HR, Bangalore S, O'Brien SM, Boden WE, et al. Initial Invasive or Conservative Strategy for Stable Coronary Disease. New England Journal of Medicine. 2020; 382: 1395-1407.

9. Reeh J, Therming CB, Heitmann M, Hojberg S, Sorum C, Bech J, et al. Prediction of obstructive coronary artery disease and prognosis in patients with suspected stable angina. European Heart Journal. 2019; 40: 1426-1435.

10. Arnold SV, Spertus JA, Ciechanowski PS, Soine LA, Jordan-Keith K Caldwell JH, et al. Psychosocial modulators of angina response to myocardial ischemia. Circulation. 2009; 120: 126-133.

11. Knuuti J, Wijns W, Achenbach S, Agewall S, Barbato E, Bax JJ, et al. 2019 ESC guidelines for the diagnosis and management of chronic coronary syndromes. European Heart Journal. Oxford University Press. 2020; 41: 407477.

12. Agrawal S, Mehta PK, Bairey Merz CN. Cardiac Syndrome X: Update 2014 Cardiology Clinics. W.B. Saunders. 2014; 32: 463-478.
13. Gould KL, Johnson NP. Coronary Physiology Beyond Coronary Flow Reserve in Microvascular Angina: JACC State-of-the-Art Review. Journal of the American College of Cardiology. Elsevier USA. 2018; 72: 2642-2662.

14. Hoffman JIE, Buckberg GD. Pathophysiology of Subendocardial Ischaemia. BMJ. 1975; 1: 76-79.

15. Salazar CA, Basilio Flores JE, Veramendi Espinoza LE, Mejia Dolores JW, Rey Rodriguez DE, Loza Munárriz C. Ranolazine for stable angina pectoris. Cochrane Database of Systematic Reviews. John Wiley and Sons Ltd. 2017; 2017.

16. Rayner-Hartley E, Parvand M, Humphries $\mathrm{KH}$, Starovoytov A, Park JE, Sediak T. Ranoazine for Symptomatic Management of Microvascular Angina. Am J Ther. 2020; 27.

17. Knuuti J, Wijns W, Saraste A, Capodanno D, Barbato E, et al. 2019 ESC Guidelines for diagnosis and management of chronic coronary syndromes: The Task Force for the diagnosis and management of chronic coronary syndromes of the European Society of Cardiology (ESC). Eur Heart J. 2020; 41: 407-477.

18. Hirai T, Aaron Grantham J, Sapontis J, Cohen DJ, Marso SP, Lombardi W, et al. Quality of life changes after chronic total occlusion angioplasty in patients with baseline refractory angina. Circulation: Cardiovascular Interventions. $2019 ; 12$.

19. Mannheimer C, Carlsson CA, Ericsson K, Vedin A, Wilhelmsson C. Transcutaneous electrical nerve stimulation in severe angina pectoris. European Heart Journal. 1982; 3: 297-302.

20. Hallén K, Hrafnkelsdóttir T, Jern S, Biber B, Mannheimer C, Roy SD. Transcutaneous electrical nerve stimulation induces vasodilation in healthy controls but not in refractory angina patients. Journal of Pain and Symptom Management. 2010; 40: 95-101.

21. Holland LC, Navaratnarajah M, Taggart DP. Does surgical sympathectomy improve clinical outcomes in patients with refractory angina pectoris? Interactive Cardiovascular and Thoracic Surgery. 2016; 22: 488-492.

22. Tan Z, Nalpon J, Valchanov K. Case Series of Left Stellate Ganglion Blocks for Refractory Angina Pectoris: 14 year later and still efficacious. Journal of Pain and Symptom Management. 2019; 58: 11-14.

23. Lanza GA, Grimaldi R, Greco S, Ghio S, Sarullo F, Zuin G. Spinal cord stimulation for the treatment of refractory angina pectoris: a multicenter randomized single-blind study (the SCS-ITA trial). Pain. 2011; 152: 45-52.

24. Gomes B, Valchanov K, Davies W, Borwn A, Schofield P. Spinal cord stimulation for refractory angina: 100 case-experience from the National Refractory Angina Service. British J Cardiol. 2016; 23: 106-109.

25. Pan X, Bao H, Si Y, Chen H, Gao X, Xie X, et al. Spinal Cord Stimulation for Refractory Angina Pectoris: A Systematic Review and Meta-analysis. Clin J Pain. 2017; 33.

26. Task Force Members, Montalescot G, Sechtem U, Achenbach S, Andreotti F, Arden C. 2013 ESC guidelines on the management of stable coronary artery disease: The Task Force on the management of stable coronary artery disease of the European Society of Cardiology. Eur Heart J. 2013; 34: 29493003.

27. Chakravarthy K, Malayil R, Kirketeig T, Deer T. Burst Spinal Cord Stimulation: A Systematic Review and Pooled Analysis of Real-World Evidence and Outcomes Data. Neuromodulation. 2018; 21: 10-18.

28. Asbury EA, Webb CM, Probert H, Wright C, Barbir M, Fox K, et al. Cardiac rehabilitation to improve physical functioning in refractory angina: A pilot study. Cardiology (Switzerland). 2012; 122: 170-177.

29. Moore RK, Groves GG, Bridson JD, Grayson AD, Wong H, et al. A brief cognitive-behavioral intervention reduces hospital admissions in refractory angina patients. J Pain Symptom Manage. 2007; 33: 310-316.

30. Lawson WE, Hui JCK, Kennard ED, Linnemeier G. Enhanced External Counterpulsation is Cost-Effective in Reducing Hospital Costs in Refractory Angina Patients. Clinical Cardiology. 2015; 38: 344-349.

31. Martinez-Sanchez C, Azar-Manzur F, Gonzales-Pacheto H, Amezcua-Guerra 
LM, Masso $F$, et al. Effectiveness and safety of extracorporeal shockwave myocardial revascularization in patients with refractory angina pectoris and heart failure. Am Col Cardiol. 2021; 144: 26-32.

32. Briones E, Lacalle JR, Marin-Leon I, Rueda JR. Transmyocardial lase revascularization versus medical therapy for refractory angina. Cochrane Database Syst Rev. 2015; 2015.

33. Bazoukis G, Brilakis ES, Tse G, Letsas KP, Kitsoulis P, Liu T, et al. The efficacy of coronary sinus reducer in patients with refractory angina-A systematic review of the literature. Journal of Interventional Cardiology. 2018; 31: $775-779$
34. D'Amico G, Giannini F, Massuisi M, Tebaldi M, Cafaro A, et al. Usefulness of coronary sinus reducer implantation for the treatment of chronic refractory angina pectoris. Am J Cardiol. 2021; 139: 22-27.

35. Hartikainen J, Hassinen I, Hedman A, Kivelä A, Saraste A, Knuuti J, et al. Adenoviral intramyocardial VEGF-DDNDC gene transfer increases myocardial perfusion reserve in refractory angina patients: A phase I/Ila study with 1-year follow-up. European Heart Journal. 2017; 38: 2547-2555.

36. Shah R, Latham SB, Khan SA, Shahreyar M, Hwang I, Jovin IS. A comprehensive meta-analysis of stem cell therapy for chronic angina. Clinical Cardiology. 2018; 41: 525-531. 